\title{
Central nervous system tumor registration in the Swedish Cancer Register and Inpatient Register between 1990 and 2014
}

This article was published in the following Dove Press journal:

Clinical Epidemiology

\author{
Giorgio Tettamanti' \\ Rickard Ljung' \\ Anders Ahlbom' \\ Mats Talbäck' \\ Birgitta Lannering ${ }^{2}$ \\ Tiit Mathiesen ${ }^{3,4}$ \\ Jenny Pettersson Segerlind ${ }^{3}$ \\ Maria Feychting' \\ 'Unit of Epidemiology, Institute of \\ Environmental Medicine, Karolinska \\ Institutet, Stockholm, Sweden; \\ 2Pediatric Oncology, Department \\ of Clinical Sciences, University \\ of Gothenburg, Gothenburg, \\ Sweden; ${ }^{3}$ Department of Clinical \\ Neuroscience, Karolinska Institutet, \\ Stockholm, Sweden; ${ }^{4}$ Department \\ of Clinical Medicine, University of \\ Copenhagen and Rigshospitalet, \\ Copenhagen, Denmark
}

Correspondence: Giorgio Tettamanti Unit of Epidemiology, Institute of Environmental Medicine, Karolinska Institutet, Nobels väg 13, 17165 Solna, Stockholm, Sweden

Email giorgio.tettamanti@ki.se
Background: The Swedish Cancer Register (SCR) is characterized by excellent quality and completeness overall, but the quality of the reporting may vary according to tumor site and age, and may change over time. The aim of the current study was to investigate the completeness of the reporting of central nervous system (CNS) tumor cases to the SCR.

Materials and methods: Individuals hospitalized for a CNS tumor between 1990 and 2014 were identified using the Inpatient Register; the proportion of identified cases that did not have any cancer diagnosis reported to the SCR was subsequently assessed.

Results: Between 1990 and 2014, 58,698 individuals were hospitalized for a CNS tumor, and a large proportion of them did not have any cancer diagnosis reported to the SCR (26\%). This discrepancy was particularly pronounced for benign tumors and among elderly patients (over $30 \%)$. It was substantially lower for malignant brain tumors among adults (10\%); moreover, no increase in the discrepancy between the two registers was observed in this group during the study period. Similar findings were found when assessing the concordance between the Cause of Death Register and the SCR. Among CNS tumor patients who were not reported to the SCR, a large proportion had only one hospital discharge diagnosis containing a CNS tumor (35\%) and were less likely to be found in the Outpatient Register, which indicates that a large proportion of patients may have received an erroneous diagnosis.

Conclusion: While a large proportion of CNS tumor patients were not reported to the SCR, the discrepancy between the SCR and the Inpatient Register was relatively small for malignant brain tumors among adults and has remained stable throughout the study period. We do not recommend that data from the Inpatient Register are combined with the SCR to estimate CNS tumor incidence, without proper confirmation of the diagnoses, as a considerable proportion of CNS tumor diagnoses registered in the Inpatient Register is unlikely to reflect true CNS tumors.

Keywords: brain neoplasms, central nervous system neoplasms, registries, Sweden

\section{Introduction}

The Swedish Cancer Register (SCR) is an extremely valuable source of information for conducting epidemiological research. The SCR was established in 1958 and contains information about all malignant tumors and certain benign tumors (such as benign brain tumors), diagnosed in Sweden. According to Swedish act and government ordinance, reporting of newly diagnosed primary cancer cases to the SCR is statutory for all healthcare providers.

Overall, the SCR has an excellent quality and completeness: a validation study estimated that, in $1998, \sim 96 \%$ of the newly diagnosed cancer cases were reported to the SCR. ${ }^{1}$ However, the study also reported that the quality of the reporting was site 
and age specific. While the completeness was high for breast, female genital organs, and urologic sites, considerable underreporting was observed for leukemia, lymphoma, soft tissue, and nervous system tumors. Moreover, the underreporting was more elevated among elderly patients and for diagnoses that were not histologically confirmed. ${ }^{1}$

Two recent studies have investigated the underreporting of pancreatic cancer to the SCR: both the studies reported that pancreatic cancer cases were largely underreported. ${ }^{2,3}$ Moreover, a recent Swedish study has shown that $\sim 40 \%$ of liver cancer patients was not reported to the SCR. ${ }^{4}$ Findings from these studies indicate that for certain cancer diagnoses there could be substantial underreporting, especially if these cancers are associated with relatively short survival, as it is for pancreatic and liver cancer and tumors of the central nervous system (CNS).

The incidence of CNS tumors in Sweden has been stable during the last 40 years, but with some differences between tumor subtypes and age groups. ${ }^{5,6}$ Incidence trend studies describe potential changes in the occurrence of specific tumors over time, and although population exposure is at an ecological level, they have been useful, for example, in checking the plausibility of reported increased risks of glioma associated with mobile phone use. ${ }^{6-11}$ However, such analyses rely on the complete reporting of tumor occurrences to the cancer registers; if completeness of reporting changes over time, spurious changes of the incidence may be observed, or an increase in incidence may go undetected. According to Kilander et al the observed declining incidence trends of pancreatic cancer ${ }^{12-14}$ could be caused by changes in the underreporting to the cancer registers. ${ }^{3}$ Moreover, if the underreporting of CNS tumor diagnoses to the SCR is substantial, it may affect the statistical power of epidemiological studies on CNS tumor etiology and may introduce selection bias in studies relying solely on cancer register information for identification of cases. Therefore, it is of extreme importance, for both incidence trend and etiological studies, to evaluate the completeness of CNS tumor reporting to the SCR.

Therefore, the aim of the current study was to assess the completeness of the reporting of CNS tumors to the Swedish Cancer Register by estimating the concordance of the SCR with the Inpatient Register between 1990 and 2014.

\section{Methods}

\section{Identification of CNS tumor patients}

We identified all hospital discharges that included a CNS tumor diagnosis, using the Swedish Inpatient Register. ${ }^{15}$ Reporting of hospital discharge diagnoses to the Inpatient
Register is mandatory for all publicly and privately funded healthcare providers. The Inpatient Register became nationwide in 1987, and the coverage is $\sim 99 \%$. The accuracy for most of the diagnostic codes varies between $85 \%$ and $95 \%$. ${ }^{15}$ All first occurrences of a CNS tumor diagnosis in the Inpatient Register between 1987 and 2014 were extracted and, to exclude prevalent CNS tumor cases, individuals who had a diagnosis between 1987 and 1989 were excluded. CNS tumor patients were first divided into brain tumors and other CNS tumors and were then further classified according to tumor behavior (malignant, benign, and unknown). Since the Inpatient Register does not contain information on morphology, it was not possible to investigate more specific tumor subtypes.

The SCR was used to identify all CNS tumor diagnoses that were reported between 1990 and 2015 and to collect information about all other cancer diagnoses for the patients hospitalized with a CNS tumor diagnosis. Some of the CNS tumors identified in the Inpatient Register are likely to be metastases from other primary tumors and should not be reported to the SCR. Therefore, we defined CNS tumor patients identified in the Inpatient Register as "not found in the SCR" if they did not have any cancer diagnosis in the SCR at any point in time (ie, between 1958 and 2015): this discrepancy between the two registers is the main focus of the study. In a secondary analysis, the Swedish Cause of Death Register was used to identify individuals who had a CNS tumor as main or contributing cause of death. ${ }^{16}$ The ninth and tenth revisions of the ICD were used to identify individuals with a CNS tumor diagnosis in the Inpatient and Cause of Death Registers during the study period. Brain tumors were defined as ICD10 codes C71, D33.0-D33.2, D43.0-D43.2, while other CNS tumors were defined as ICD10 codes C70, C72, D32, D33.3-D33.9, D42, D43.3-D43.9. In this study, tumors of the pineal and pituitary glands and craniopharyngeal duct were not regarded as CNS tumors, although in some instances, such as in NORDCAN, they are considered as CNS tumors. ${ }^{17}$ Corresponding codes for the time period covered by ICD9 were used (see Table S1 for exact definition). The unique personal identity number provided to all Swedish residents have been used to link data from the three registers. The study was approved by the Regional Ethical Review Board in Stockholm (2011/634-31/4).

\section{Statistical analysis}

As the main analysis, the discrepancy between the Inpatient Register and the SCR was evaluated (divided into brain tumors and other CNS tumors) according to tumor behavior (malignant, benign, and unknown), period of hospitalization 
(1990-1999, 2000-2009, and 2010-2014), and whether the CNS tumor was the main or a secondary diagnosis in the Inpatient Register. Moreover, we further evaluated this discrepancy among patients who had a cranial surgery.

In order to estimate the proportion of patients who may have had an erroneous CNS tumor diagnosis in the Inpatient Register, sensitivity analyses were performed in which the discrepancy between the two registers was evaluated according to number of hospitalizations and type of clinic in which the patients had been hospitalized. We also checked whether CNS tumor cases that were not reported to the SCR had a CNS tumor diagnosis in the Outpatient Register: this analysis was performed only for patients who survived at least 1 year (to avoid including patients unlikely to be treated in outpatient care because of the severity of their disease, which could have prevented discharge from hospital care and is associated with short cancer survival), and were diagnosed from 2001 onward, the year in which the Outpatient Register was established. Individuals who migrated from or immigrated to Sweden before the date of the CNS tumor diagnosis in the Inpatient Register, and did not have any cancer diagnosis in the SCR, were excluded from the analysis: this was done to remove individuals who might have had the cancer diagnosis abroad and were hospitalized and treated in Sweden for a prevalent cancer. In order to determine whether the reporting to the SCR was related with short-term survival and, consequently, tumor severity, Cox regression models were used to compare the survival of CNS tumor patients reported to the SCR compared to those who were not reported. In the Cox regression models, age was used as the underlying time scale, and analyses were adjusted for year of hospitalization and location of the CNS tumor (brain tumor/other CNS tumor): analyses were stratified by tumor behavior and age at first hospitalization (age $<70$ years; age $\geq 70$ years). All statistical analyses were performed using Stata 14.2 (StataCorp LP, College Station, TX, USA).

\section{Results}

Between 1990 and 2014, 58,698 individuals had been hospitalized with a CNS tumor diagnosis: $50 \%$ of them had a CNS tumor diagnosis reported to the SCR during the same time period ( $n=29335)$, while $26 \%$ of them did not have any cancer diagnosis reported to the SCR at any point in time $(\mathrm{n}=15,100)$. Among the 14,263 CNS tumor patients who did not have a CNS tumor in the SCR but had instead another cancer diagnosis reported to the SCR, the most common diagnoses were lung cancer $(13 \%)$, breast cancer $(11 \%)$, and prostate cancer (7\%). There were more than 30,000 patients who had a CNS tumor diagnosis reported to the SCR $(n=30,728)$ and more than $95 \%$ of them were found also in the Inpatient Register $(n=29,335)$.

The discrepancy between the two registers was lower for brain tumors (22\%), compared to other CNS tumors (31\%), and it was particularly elevated for benign tumors (Table 1). Sensitivity analyses that took into account the number of hospital discharges containing a CNS tumor diagnosis showed that the discrepancies decreased with the number of hospitalizations (Table S2). Moreover, a high proportion of patients had been hospitalized only once for a CNS tumor (35\%). The types of clinics in which hospitalized patients got a CNS tumor diagnosis were mainly internal medicine, neurosurgery, and neurology clinics (44\%, 34\%, and $16 \%$ respectively) (not shown). The proportion of CNS tumor patients identified in the Inpatient Register that was not found in the SCR was lower if the CNS tumor patient was treated at a neurosurgery clinic (15\%) compared to the other two clinics ( $22 \%$ for neurology and $27 \%$ for internal medicine).

CNS tumor patients who had the CNS tumor as the main diagnosis in the Inpatient register were more likely to have a cancer diagnosis in the SCR compared to patients who had the CNS tumor as a secondary diagnosis (Table 2). Moreover, brain tumor patients who had a cranial surgery were substantially more likely to be found in the SCR: only $3 \%$ of patients with a malignant brain tumor who had a cranial surgery did not have any cancer diagnosis reported to the SCR, while over a third of the patients who did not have a cranial surgery were not found in the SCR (Table 2).

\section{Analysis stratified by age at CNS tumor diagnosis}

The discrepancy between the Inpatient Register and the SCR differed according to age at diagnosis (Table 1). CNS tumor patients who did not have any cancer diagnosis reported to the SCR were older than those reported: the age difference was particularly pronounced for those with a malignant CNS tumor. While only a small proportion of children with a malignant CNS tumor had no cancer diagnosis in the SCR $(\sim 10 \%)$, this proportion was substantially higher among elderly individuals (Table 1). For benign tumors and tumors with unknown behavior, the discrepancy between the two registers was particularly elevated also among children and adolescents (Table 1). 
Table I Total number and proportions of central nervous system tumor cases in the Inpatient Register not found in the Swedish Cancer Register by age at first hospitalization between 1990-2014

\begin{tabular}{|c|c|c|c|c|c|c|c|c|}
\hline \multicolumn{9}{|l|}{ Brain tumors } \\
\hline \multirow{2}{*}{$\begin{array}{l}\text { Age at } \\
\text { hospitalization } \\
\text { (years) }\end{array}$} & \multicolumn{2}{|c|}{ All combined } & \multicolumn{2}{|l|}{ Malignant } & \multicolumn{2}{|l|}{ Benign } & \multicolumn{2}{|c|}{ Unknown behavior } \\
\hline & $\begin{array}{l}\text { Not } \\
\text { reported, } \\
\text { n (\%) }\end{array}$ & Total & $\begin{array}{l}\text { Not } \\
\text { reported, } \\
\text { n (\%) }\end{array}$ & Total & $\begin{array}{l}\text { Not } \\
\text { reported, } \\
\text { n (\%) }\end{array}$ & Total & $\begin{array}{l}\text { Not } \\
\text { reported, } \\
\text { n (\%) }\end{array}$ & Total \\
\hline $0-19$ & $491(20.3)$ & 2,414 & $120(9.0)$ & 1,329 & $12 \mid(34.9)$ & 347 & $251(33.3)$ & 754 \\
\hline $20-69$ & $2,994(15.3)$ & 19,630 & $910(9.6)$ & 9,486 & 69I (34.3) & 2,014 & $1,399(17.0)$ & 8,237 \\
\hline $70+$ & $4,214(33.6)$ & 12,565 & $2,165(32.7)$ & 6,629 & $286(40.5)$ & 706 & $\mathrm{I}, 784(33.8)$ & 5,286 \\
\hline Overall & $7,699(22.2)$ & 34,612 & $3,195(18.3)$ & 17,444 & I,098 (35.8) & 3,067 & $3,434(24.0)$ & 14,277 \\
\hline $\begin{array}{l}\text { Mean age at } \\
\text { hospitalization }\end{array}$ & 64.0 & 59.0 & 70.0 & 59.5 & 50.4 & 50.6 & 62.7 & 60.2 \\
\hline \multicolumn{9}{|c|}{ Other central nervous system tumors (excluding brain tumors) } \\
\hline $0-19$ & $199(35.7)$ & 557 & $17(10.1)$ & 169 & $122(52.1)$ & 234 & $60(38.0)$ & 158 \\
\hline $20-69$ & $3,259(22.9)$ & 14,206 & $110(9.7)$ & 1,134 & $2,637(23.5)$ & 11,230 & $512(27.7)$ & 1,850 \\
\hline $70+$ & $3,943(42.3)$ & 9,323 & $208(25.6)$ & 813 & $3,310(44.8)$ & 7,394 & $430(38.1)$ & $\mathrm{I}, \mathrm{I} 30$ \\
\hline Overall & $7,40 \mathrm{I}(30.7)$ & 24,086 & $335(15.8)$ & 2,116 & $6,069(32.2)$ & 18,858 & $1,002(32.0)$ & 3,138 \\
\hline $\begin{array}{l}\text { Mean age at } \\
\text { hospitalization }\end{array}$ & 66.5 & 62.1 & 67.5 & 59.1 & 67.5 & 62.9 & 60.4 & 59.8 \\
\hline
\end{tabular}

Table 2 Total number and proportions of central nervous system tumor cases in the Inpatient Register not found in the Swedish Cancer Register by diagnosis (main/secondary) and cranial surgery (yes/no) between 1990-2014

\begin{tabular}{|c|c|c|c|c|c|c|c|c|}
\hline \multicolumn{9}{|l|}{ Brain tumors } \\
\hline & \multicolumn{2}{|c|}{ All combined } & \multicolumn{2}{|l|}{ Malignant } & \multicolumn{2}{|l|}{ Benign } & \multicolumn{2}{|c|}{ Unknown behavior } \\
\hline & $\begin{array}{l}\text { Not } \\
\text { reported, } \\
\text { n (\%) } \\
\end{array}$ & Total & $\begin{array}{l}\text { Not } \\
\text { reported, } \\
\text { n (\%) }\end{array}$ & Total & $\begin{array}{l}\text { Not } \\
\text { reported, } \\
\text { n (\%) }\end{array}$ & Total & $\begin{array}{l}\text { Not } \\
\text { reported, } \\
\text { n (\%) }\end{array}$ & Total \\
\hline Main diagnosis & $5,950(20.8)$ & 28,619 & $2,717(18.1)$ & 14,993 & $719(32.5)$ & 2,211 & $2,534(21.9)$ & 11,552 \\
\hline $\begin{array}{l}\text { Secondary } \\
\text { diagnosis }\end{array}$ & I,749 (29.2) & 5,993 & 478 (19.5) & 2,451 & $379(44.3)$ & 856 & $900(33.0)$ & 2,725 \\
\hline Cranial surgery & I,082 (7.I) & 15,435 & $244(3.0)$ & 8,016 & $44 \mid(24.6)$ & 1,796 & $40 I(7.0)$ & 5,691 \\
\hline $\begin{array}{l}\text { No cranial } \\
\text { surgery }\end{array}$ & $6,617(34.5)$ & 19,177 & $2,951(31.3)$ & 9,428 & $657(51.7)$ & 1,271 & $3,033(35.3)$ & 8,586 \\
\hline \multicolumn{9}{|c|}{ Other central nervous system tumors (excluding brain tumors) } \\
\hline Main diagnosis & $3,787(22.3)$ & 16,998 & $204(15.3)$ & 1,330 & $2,902(21.9)$ & 13,284 & $685(28.5)$ & 2,406 \\
\hline $\begin{array}{l}\text { Secondary } \\
\text { diagnosis }\end{array}$ & $3,614(51.0)$ & 7,088 & $131(16.7)$ & 786 & $3,167(56.8)$ & 5,574 & $317(43.3)$ & 732 \\
\hline Cranial surgery & $1,212(12.4)$ & 9,788 & $9(2.8)$ & 322 & $1,113(12.7)$ & 8,750 & $90(12.5)$ & 721 \\
\hline $\begin{array}{l}\text { No cranial } \\
\text { surgery }\end{array}$ & $6,189(43.3)$ & 14,298 & $326(18.2)$ & 1,794 & $4,956(49.0)$ & 10,108 & $912(37.7)$ & 2,417 \\
\hline
\end{tabular}

\section{Analysis stratified by calendar period}

As shown in Figure 1, the discrepancy between the Inpatient Register and the SCR for brain tumors increased slightly during the first years of the 1990s, remained rather constant between 1996 and 2009, after which it has decreased. In 2014 the discrepancy was almost back at the level observed in 1990 (21\% compared to $19 \%)$. However, for benign brain tumors, it increased during the second decade and remained at a high level until the end of the study period. For malignant brain tumors, as well as for those with unknown behavior, a decrease was observed since the beginning of the 2000s, particularly among individuals aged 20-69 years (Figure 1; Table 3). Among elderly individuals diagnosed with a malignant brain tumor, a small increase in the discrepancy 
between the two registers was observed in the second decade (+4\%): however, after 2010 it decreased by $10 \%$ (Table 3 ). The discrepancy for malignant brain tumors among children and adolescents has constantly increased over time, by $\sim 3 \%$ in each decade (Table 3 ). For other CNS tumors the proportion of cases identified in the Inpatient Register that was not found in the SCR has increased from $25 \%$ in 1990 to $33 \%$ in 2014: this increase was mainly driven by the discrepancies observed for benign tumor patients, particularly from 2004, which however leveled after 2008 (Figure 2).

\section{Survival analysis}

Age-specific Cox regression analyses showed that elderly CNS tumor patients (age $\geq 70$ years) diagnosed with a benign or malignant tumor, who had no cancer diagnosis in the SCR, had a worse survival compared to those reported; whereas

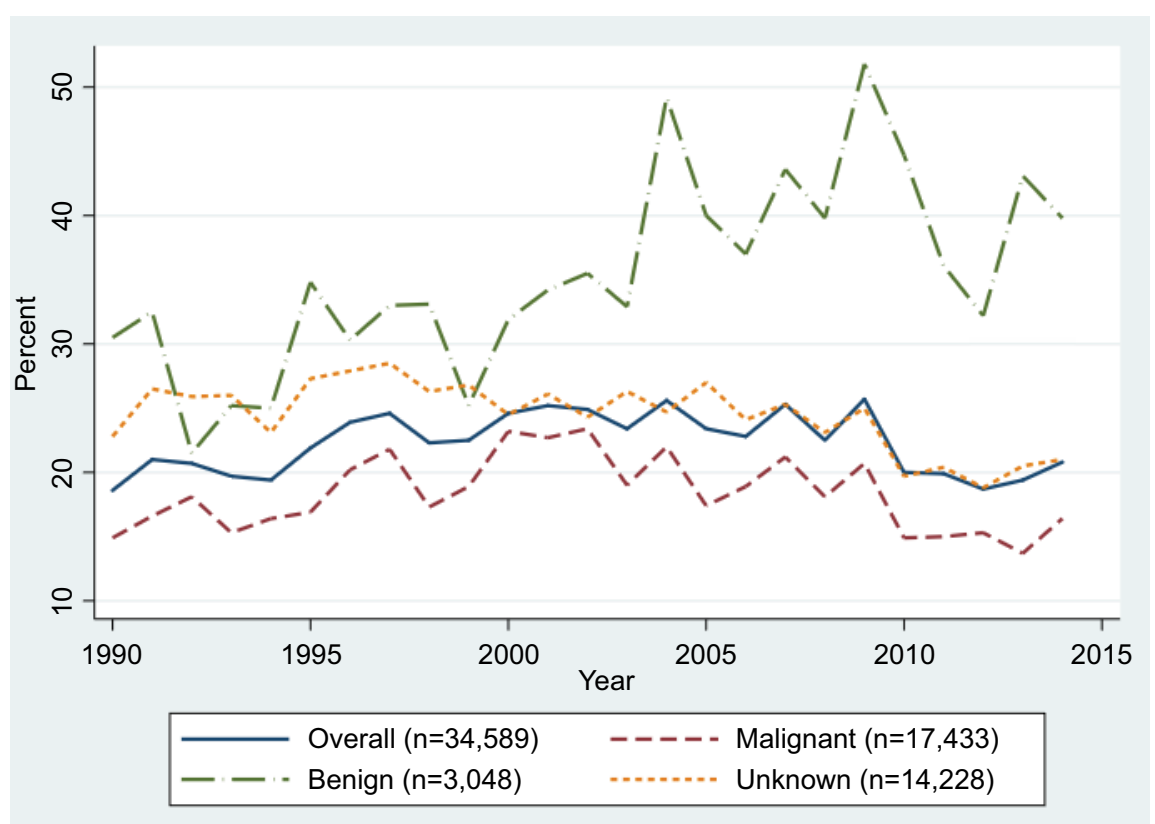

Figure I Proportions of brain tumor cases in the Inpatient Register not found in the Swedish Cancer Register by tumor behavior between $1990-2014$.

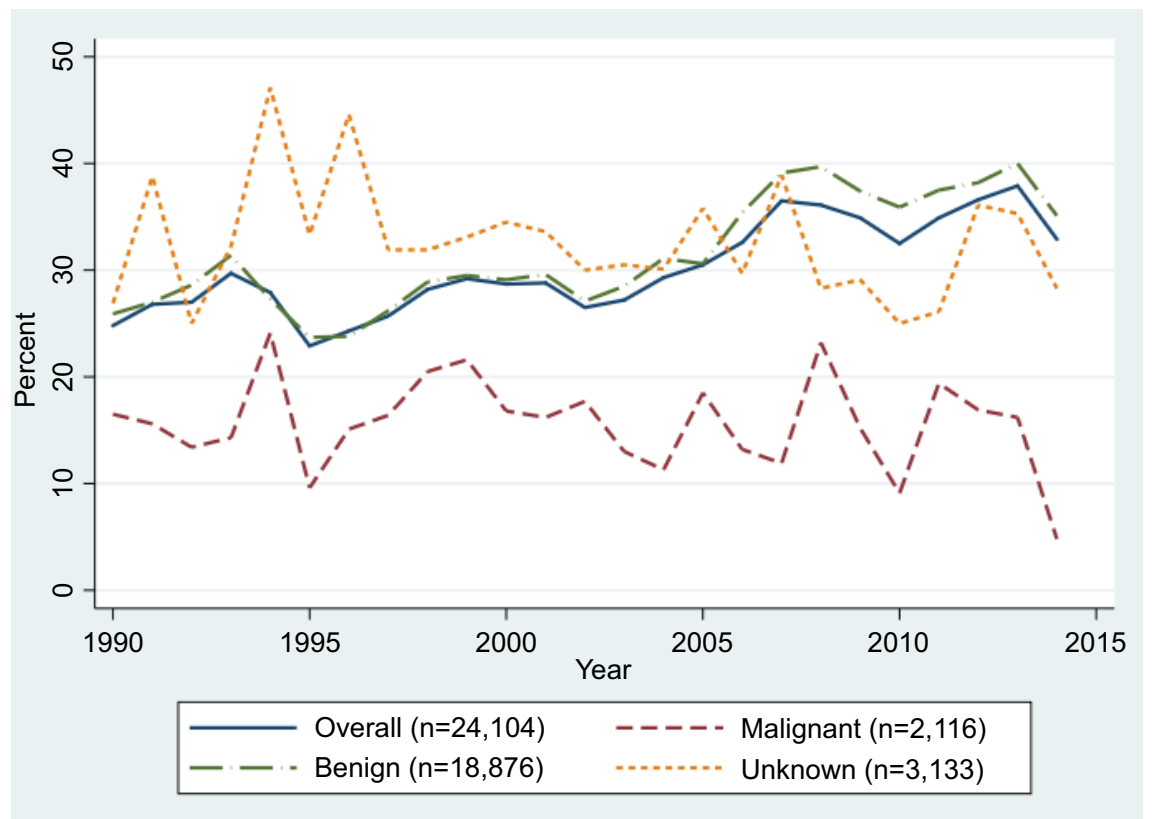

Figure 2 Proportions of other central nervous system tumor cases in the Inpatient Register not found in the Swedish Cancer Register by tumor behavior between $1990-2014$. 
Table 3 Proportions of central nervous system tumor cases in the Inpatient Register not found in the Swedish Cancer Register between 1990-2014 by age at first hospitalization and decade of diagnosis

\begin{tabular}{|c|c|c|c|c|c|c|c|c|}
\hline \multicolumn{9}{|l|}{ Brain tumors } \\
\hline \multirow[b]{2}{*}{$\begin{array}{l}\text { Age at } \\
\text { hospitalization } \\
\text { (years) } \\
\text { Between } \\
1990-1999\end{array}$} & \multicolumn{2}{|c|}{ All combined } & \multicolumn{2}{|l|}{ Malignant } & \multicolumn{2}{|l|}{ Benign } & \multicolumn{2}{|c|}{ Unknown behavior } \\
\hline & $\begin{array}{l}\text { Not } \\
\text { reported, } \\
\text { n (\%) }\end{array}$ & Total & $\begin{array}{l}\text { Not } \\
\text { reported, } \\
\text { n (\%) }\end{array}$ & Total & $\begin{array}{l}\text { Not } \\
\text { reported, } \\
\text { n (\%) }\end{array}$ & Total & \begin{tabular}{|l} 
Not \\
reported, \\
n (\%)
\end{tabular} & Total \\
\hline $0-19$ & $162(16.4)$ & 989 & $39(6.8)$ & 578 & $34(23.5)$ & 145 & $89(32.3)$ & 276 \\
\hline $20-69$ & $\mathrm{I}, 067(\mathrm{I} 4.2)$ & 7,536 & $417(9.8)$ & 4,262 & $172(25.6)$ & 672 & $481(18.1)$ & 2,661 \\
\hline $70+$ & $1,619(34.2)$ & 4,730 & $893(32.0)$ & 2,794 & $97(40.9)$ & 237 & $639(37.0)$ & $\mathrm{I}, 727$ \\
\hline Overall & $2,848(21.5)$ & 13,255 & I,349 (I7.7) & 7,634 & $303(28.8)$ & 1,054 & $1,209(25.9)$ & 4,664 \\
\hline \multicolumn{9}{|l|}{$\begin{array}{l}\text { Between } \\
2000-2009\end{array}$} \\
\hline $0-19$ & $211(23.0)$ & 919 & $47(9.8)$ & 482 & $67(43.8)$ & 153 & 98 (33.9) & 289 \\
\hline $20-69$ & $1,287(16.5)$ & 7,798 & $370(10.4)$ & 3,557 & $344(39.0)$ & 882 & $576(17.0)$ & 3,383 \\
\hline $70+$ & $\mathrm{I}, 874(36.4)$ & 5,145 & $956(36.5)$ & 2,662 & $119(40.2)$ & 296 & $809(36.0)$ & 2,249 \\
\hline Overall & $3,372(24.3)$ & 13,862 & $\mathrm{I}, 373(20.5)$ & 6.701 & $530(39.8)$ & 1,331 & $\mathrm{I}, 483(25.0)$ & 5,921 \\
\hline \multicolumn{9}{|l|}{ Between } \\
\hline $0-19$ & $118(23.3)$ & 506 & $34(12.6)$ & 269 & $20(40.8)$ & 49 & 64 (33.9) & 189 \\
\hline $20-69$ & $640(14.9)$ & 4,296 & $123(7.4)$ & 1,667 & $175(38.0)$ & 460 & $342(15.6)$ & 2,193 \\
\hline $70+$ & $721(26.8)$ & 2,690 & $316(26.1)$ & 1.213 & $70(40.5)$ & 173 & $336(25.7)$ & 1,310 \\
\hline Overall & $\mathrm{I}, 479(19.7)$ & 7,492 & $473(15.0)$ & 3,149 & $265(38.9)$ & 682 & $742(20.1)$ & 3,692 \\
\hline \multicolumn{9}{|c|}{ Central nervous system tumors (excluding brain tumors) } \\
\hline \multicolumn{9}{|l|}{ Between } \\
\hline $1990-1999$ & & & & & & & & \\
\hline $0-19$ & $87(34.5)$ & 252 & $9(9.7)$ & 92 & $63(52.9)$ & 119 & 15 (35.7) & 42 \\
\hline $20-69$ & $909(18.9)$ & 4,805 & $49(10.5)$ & 465 & 740 (I8.8) & 3,922 & $120(28.4)$ & 422 \\
\hline $70+$ & $1,114(39.3)$ & 2,838 & $86(29.2)$ & 295 & $925(40.2)$ & 2,303 & $104(42.3)$ & 246 \\
\hline Overall & $2,110(26.7)$ & 7,895 & I44 (I6.9) & 852 & $\mathrm{I}, 728(27.4)$ & 6,344 & \begin{tabular}{|l|}
$239(33.7)$ \\
\end{tabular} & 710 \\
\hline \multicolumn{9}{|l|}{ Between } \\
\hline $0-19$ & 7I (35.2) & 202 & $7(13.0)$ & 54 & $32(43.8)$ & 73 & $32(41.0)$ & 78 \\
\hline $20-69$ & $1,386(23.3)$ & 5,955 & $39(8.2)$ & 474 & $\mathrm{I}, \mathrm{I}$ II (24.2) & 4,599 & $235(26.6)$ & 884 \\
\hline $70+$ & $\mathrm{I}, 720(43.2)$ & 3,979 & $99(25.5)$ & 389 & $\mathrm{I}, 386(46.4)$ & 2,987 & $239(39.1)$ & 611 \\
\hline Overall & $3,177(31.3)$ & 10,136 & $145(15.8)$ & 917 & $2,530(33.0)$ & 7,659 & $763(31.4)$ & 2,428 \\
\hline \multicolumn{9}{|l|}{ Between } \\
\hline $0-19$ & $4 \mid(39.8)$ & 103 & I (4.3) & 23 & $27(64.3)$ & 42 & $13(34.2)$ & 38 \\
\hline $20-69$ & $964(28.0)$ & 3,446 & $22(11.3)$ & 195 & $785(29.0)$ & 2,709 & $157(29.0)$ & 542 \\
\hline $70+$ & I, $109(44.3)$ & 2,506 & $23(17.8)$ & 129 & 999 (47.5) & 2,104 & 87 (31.9) & 273 \\
\hline Overall & $2,114(34.9)$ & 6055 & $46(13.3)$ & 347 & $\mathrm{I}, 8 \mathrm{II}(37.3)$ & 4,855 & $257(30.1)$ & 855 \\
\hline
\end{tabular}

among younger patients (age $<70$ years), those who were not found in the SCR had instead a better survival (Table 4).

\section{Concordance between Inpatient Register and Outpatient Register}

When examining the concordance between the Inpatient and Outpatient Registers, we found that CNS tumor patients who did not have any cancer diagnosis reported to the SCR were also less likely to have a CNS tumor diagnosis in the Outpatient Register, compared to patients who were found in the SCR. For example, 36\% of brain tumor cases not found in the SCR had no CNS tumor diagnosis in the Outpatient Register, while only $15 \%$ of the brain tumor cases found also in the SCR did not have a CNS tumor reported to the Outpatient Register. Similar findings were found in analysis stratified by age at diagnosis (Table S3). 
Table 4 Five-year and overall mortality after central nervous tumor diagnosis in the Inpatient Register according to the reporting to the Swedish Cancer Register between 1990-2014, stratified by tumor behavior and age at first hospitalization

\begin{tabular}{|c|c|c|c|c|}
\hline & \multicolumn{2}{|c|}{ Age $<70$ years } & \multicolumn{2}{|c|}{ Age $\geq 70$ years } \\
\hline & $\mathbf{N}$ & HR (95\% Cl) & $\mathbf{N}$ & HR (95\% Cl) \\
\hline \multicolumn{5}{|l|}{ 5-year survival } \\
\hline \multicolumn{5}{|l|}{ CNS tumors with unknown grading } \\
\hline Inpatient Register only & 2,216 & $0.35(0.32-0.38)$ & 2,195 & $0.86(0.8 I-0.91)$ \\
\hline Inpatient and Cancer Register & $|0,89|$ & I (ref) & 6,368 & I (ref) \\
\hline \multicolumn{5}{|l|}{ Benign CNS tumors } \\
\hline Inpatient Register only & 3,570 & $0.98(0.85-1.14)$ & 3,589 & $1.16(1.08-1.24)$ \\
\hline Inpatient and Cancer Register & 13800 & I (ref) & 8,079 & I (ref) \\
\hline \multicolumn{5}{|l|}{ Malignant CNS tumors } \\
\hline Inpatient Register only & 1,157 & $0.73(0.68-0.79)$ & 2,373 & $1.18(1.12-1.25)$ \\
\hline Inpatient and Cancer Register & 12,118 & I (ref) & 7,442 & I (ref) \\
\hline \multicolumn{5}{|l|}{ Overall survival } \\
\hline \multicolumn{5}{|l|}{ CNS tumors with unknown grading } \\
\hline Inpatient Register only & 2,216 & $0.34(0.32-0.37)$ & 2,195 & $0.97(0.92-1.03)$ \\
\hline Inpatient and Cancer Register & $|0,89|$ & I (ref) & 6,368 & I (ref) \\
\hline \multicolumn{5}{|l|}{ Benign CNS tumors } \\
\hline Inpatient Register only & 3,570 & $0.90(0.8 \mathrm{I}-0.99)$ & 3,589 & $1.26(1.20-1.33)$ \\
\hline Inpatient and Cancer Register & 13,800 & I (ref) & 8,079 & I (ref) \\
\hline \multicolumn{5}{|l|}{ Malignant CNS tumors } \\
\hline Inpatient Register only & $\mathrm{I}, 157$ & $0.63(0.58-0.68)$ & 2,373 & $\mathrm{I} .35(\mathrm{I} .28-\mathrm{I} .4 \mathrm{I})$ \\
\hline Inpatient and Cancer Register & 12,118 & I (ref) & 7,442 & I (ref) \\
\hline
\end{tabular}

Notes: Analyses adjusted for year of hospitalization, and brain tumor diagnosis. Age was used as the underlying time scale.

Abbreviation: CNS, central nervous system.

\section{Concordance between Cause of Death Register and SCR}

In a secondary analysis, we used the Cause of Death Register to identify individuals who had a CNS tumor as a main or contributing cause of death between 1990 and 2014 ( $n=19$ 029). Overall, $28 \%$ of these individuals did not have any cancer diagnosis reported to the SCR at any point in time $(n=5,311)$. However, less than $6 \%$ of individuals who had a CNS tumor as a cause of death did not have a cancer diagnosis in the SCR or a CNS tumor diagnosis in the Inpatient Register $(n=1,116)$.

\section{Discussion}

In the current study we found that a large proportion of patients $(26 \%)$ who had a CNS tumor diagnosis registered in the Inpatient Register did not have any cancer diagnosis reported to the SCR. The discrepancy between the Inpatient Register and the SCR was lower for brain tumors (22\%) compared to other CNS tumors (31\%) and was particularly pronounced for benign CNS tumors and among elderly patients, while among children and adults diagnosed with a malignant tumor, it was substantially smaller $(\sim 10 \%)$.

Our results are in agreement with the findings from a previous validation study of the SCR. ${ }^{1}$ Similarly to what we have reported, they found that a large proportion of elderly cancer patients was not reported to the SCR. However, the authors focused only on malignant tumors and evaluated the reporting to the SCR only in 1998: for these reasons, their estimated underreporting is lower than what we have reported.

Often CNS tumors are not primary tumors but metastases and should therefore not be reported to the SCR. For this reason, we assessed whether CNS tumor patients identified in the Inpatient Register had any cancer diagnosis reported to the SCR at any point in time, rather than only investigating whether they had a CNS tumor reported to the SCR. We found that approximately half of the CNS tumor patients did not have a CNS tumor diagnosis in the SCR, while $26 \%$ did not have any cancer diagnosis reported to the SCR. This difference between the two analyses is unlikely to have been caused entirely by metastatic cases; thus, it is possible that our findings have underestimated the true discrepancies between the Inpatient Register and the SCR. On the other hand, we found that patients with only one hospital discharge diagnosis with a CNS tumor recorded were less likely to be found in the SCR than patients who had several hospital discharges mentioning a CNS tumor. Moreover, a considerable number of cases not found in the SCR were treated at clinics that do not typically treat CNS tumors, quite many (30\%) had only 
one hospital discharge diagnosis with a CNS tumor recorded, and a large proportion of patients not found in the SCR did not have a CNS tumor diagnosis in the Outpatient Register. In the latter analysis we excluded patients who died within 1 year of diagnosis to avoid including patients who might not have survived long enough to seek outpatient care, and we restricted the analyses to the years when the Outpatient Register was available (from 2001). All these findings indicate that the CNS tumor diagnosis in the Inpatient Register may have been incorrect in many instances. The previously mentioned validation study of the SCR reviewed a sample of medical records for unreported cancer cases and found that for around $20 \%$ of the records, the patients did not have a cancer. ${ }^{1}$

As already pointed out by Barlow et al, there is a misunderstanding among clinicians who believe that tumor cases should be reported to the SCR only if the diagnosis is histologically or cytologically confirmed. ${ }^{1}$ Cox regression analyses indicated that elderly patients diagnosed with a benign or malignant CNS tumor who were not found in the SCR had a worse survival. This suggests that the patient's prognosis could have had an impact on the clinician's decision to perform a histological confirmation of the tumor. Therefore, individuals with a worse prognosis (because of tumor severity, advanced age, or presence of other comorbidities) were probably less likely to receive a histological confirmation, as a severely ill patient might not benefit from the procedure necessary to have the tumor histologically confirmed, and hence less likely to be reported to the SCR. This could explain the high proportion of elderly patients identified in the Inpatient Register that were not found in the SCR, almost $50 \%$ for other benign CNS tumors and over $30 \%$ for malignant brain tumors.

Also among children and adolescents we found a large discrepancy between the SCR and the Inpatient Register for benign CNS tumors. However, when assessing the concordance between the Inpatient and the Outpatient Registers, we found that $20 \%$ of the CNS tumor patients in this age group that were not found in the SCR did not have a CNS tumor diagnosis in the Outpatient Register; this proportion was substantially smaller (4\%) if the patient had a cancer diagnosis in the SCR. This indicates that some children may have had an erroneous diagnosis reported to the Inpatient Register.

When evaluating the discrepancies between the Cause of Death Register and the SCR, we found that the proportion of CNS tumor patients not found in the SCR (28\%) was similar to the one observed in the analysis of the Inpatient Register
(26\%). An explanation for the similar findings is that often the physician who compiles the death certificate may look at the previous medical history in the medical records. In fact, $\sim 94 \%$ of individuals who had a CNS tumor as main or contributing cause of death had a CNS tumor diagnosis in the Inpatient Register.

A major strength of the current study is that the data were derived from high-quality national registers and that we were able to evaluate the discrepancies between the Inpatient Register and the SCR by age at diagnosis, tumor behavior (malignant, benign, unknown), type of diagnosis (main or secondary), and by cranial surgery. Moreover, since we had data for a relatively long period of time (from 1990 to 2014), we had the opportunity to analyze the trends of these discrepancies over time. Another strength of this study was that we were able to remove individuals who emigrated or immigrated before they were hospitalized for a CNS tumor, in order to exclude CNS tumor patients diagnosed in another country who then moved to Sweden to receive treatment: only newly detected cancers should be reported to the SCR, cancers diagnosed in other countries should not be reported.

A limitation of this study is that diagnoses in the Inpatient Register have an accuracy that ranges between $85 \%$ and $95 \% .{ }^{15}$ We found that CNS tumor patients, aged $\leq 69$ years, who were not found in the SCR, had a better survival compared to those who had a cancer diagnosis reported to the SCR; this suggests that some CNS tumor patients identified through the Inpatient Register did not actually have a CNS tumor, leading to an overestimation of the discrepancies between the two registers. This is also supported by the fact that the proportion of cases not found in the SCR varies depending on the type of clinic that made the diagnosis, suggesting that the CNS tumor diagnosis reported in the Inpatient Register may be less accurate in clinics that do not primarily treat such tumors. These diagnoses could have suspected tumors that were registered in the Inpatient Register but were not later confirmed. Moreover, not all CNS tumor patients who had a cranial surgery were reported to the SCR, which clearly indicates that some CNS tumors diagnoses were not histologically confirmed after the surgery.

The fact that not all CNS tumors are reported to the SCR does not necessarily have an effect on the validity of etiological studies, but it may affect the statistical power, introduce selection bias if the exposure of interest is related with the reasons that led to the underreporting to the SCR, and overestimate survival if CNS tumor cases with a worse prognosis are less likely to be reported. Moreover, the sus- 
pected underreporting of CNS tumors could have had an impact on the incidence trends, as it has been suggested for pancreatic cancer. ${ }^{3}$ Since the discrepancies between the Inpatient Register and the SCR for malignant brain tumors were rather stable during the study period, particularly among individuals aged 20-69 years, the incidence trends for malignant brain tumors are likely not affected by spurious changes introduced by changes in the underreporting to the SCR, with the exception of the incidence in the oldest age group ( $\geq 70$ years) which may appear to have increased as a result of better reporting to the SCR. Regarding benign CNS tumors, the discrepancy between the registers has markedly increased from 2004 and has then leveled off after 2008. The increased proportion of benign CNS tumors that were not found in the SCR could have hidden a real increase in the incidence of these tumors, but could also be due to an increased detection of smaller asymptomatic benign tumors through the higher access to MRI scanning, tumors that would previously have gone unnoticed.

We observed large discrepancies between the Inpatient Register and the SCR, particularly for benign tumors and for elderly patients. Although, as we have shown, it is possible that some diagnoses in the Inpatient Register may not have been accurate, parts of the discrepancies are probably due to a true underreporting of CNS tumor cases to the SCR. Other studies have also indicated that benign CNS tumors might be underreported, for example, a study on incidence trends of intracranial meningiomas in the Nordic countries. ${ }^{5}$ A Finnish study of 42 meningioma cases showed that approximately a third of the cases was not reported to the Finnish Cancer Register. ${ }^{18}$ Moreover, a US study found an increasing incidence of benign and borderline brain tumors that was related with the increased reporting of nonhistologically confirmed cases, suggesting that these cases were previously underreported. ${ }^{19}$

The fact that there could be different procedures regarding cancer registration in different countries, and over time, should be kept in mind when comparing incidence trends or cancer registration completeness across countries. For example, the Danish Cancer Register has since 2004 adopted new electronic reporting procedures that integrate reporting to the Cancer Register with reporting to the National Patient Register. Should a tumor reported to the Patient Register, pathology register, or Cause of Death Register despite this not be found in the Cancer Register, reminders are sent to the hospitals and the physicians who failed to report the new cancer case. ${ }^{20}$ After the introduction of these new procedures, an apparent increase in the incidence of CNS tumors was observed in Denmark, while more stable trends were found in the other Nordic countries: ${ }^{17}$ this was probably due to a decreased underreporting of CNS tumors in Denmark after 2004. Such procedures would be worthwhile to consider also in Sweden, to minimize underreporting of benign and inoperable tumors to the SCR.

\section{Conclusion}

In this study we found that a large proportion of patients registered with a CNS tumor diagnosis in the Inpatient Register did not have any cancer diagnosis reported to the SCR. The discrepancy between the registers was in some instances substantial, especially among elderly patients and for benign tumors, while for malignant CNS tumors in patients below 70 years it was smaller and did not change over time. As high levels of underreporting to the SCR have been found also for pancreatic and liver cancer, it is important to remind clinicians to report cancer cases to the SCR also when histological confirmation is not available, if the tumors can be unequivocally diagnosed through other means, such as imaging. However, we do not recommend that data from the Inpatient Register is combined with the SCR to estimate CNS tumor incidence, without proper confirmation of the diagnoses, as a considerable proportion of CNS tumor diagnoses registered in the Inpatient Register is unlikely to reflect true CNS tumors. Results from our study should be taken into consideration when performing etiological and incidence trend studies on CNS tumors using the SCR.

\section{Acknowledgments}

This work was supported by grants from the Swedish Research Council and the Swedish Cancer Society.

\section{Disclosure}

The authors report no conflicts of interest in this work.

\section{References}

1. Barlow L, Westergren K, Holmberg L, Talbäck M. The completeness of the Swedish cancer register: a sample survey for year 1998. Acta Oncol. 2009;48(1):27-33.

2. Lambe M, Eloranta S, WigertzA, Blomqvist P. Pancreatic cancer; reporting and long-term survival in Sweden. Acta Oncol. 2011;50(8):1220-1227.

3. Kilander C, Mattsson F, Ljung R, Lagergren J, Sadr-Azodi O. Systematic underreporting of the population-based incidence of pancreatic and biliary tract cancers. Acta Oncol. 2014;53(6):822-829.

4. Törner A, Stokkeland K, Svensson Å, et al. The underreporting of hepatocellular carcinoma to the cancer register and a log-linear model to estimate a more correct incidence. Hepatology. 2017;65(3):885-892.

5. Klaeboe L, Lonn S, Scheie D, et al. Incidence of intracranial meningiomas in Denmark, Finland, Norway and Sweden, 1968-1997. Int J Cancer. 2005;117(6):996-1001. 
6. Deltour I, Auvinen A, Feychting M, et al. Mobile phone use and incidence of glioma in the Nordic countries 1979-2008: consistency check. Epidemiology. 2012;23(2):301-307.

7. Little MP, Rajaraman P, Curtis RE, et al. Mobile phone use and glioma risk: comparison of epidemiological study results with incidence trends in the United States. BMJ. 2012;344:e1147.

8. Inskip PD, Hoover RN, Devesa SS. Brain cancer incidence trends in relation to cellular telephone use in the United States. Neuro Oncol. 2010;12(11):1147-1151.

9. de Vocht F, Burstyn I, Cherrie JW. Time trends (1998-2007) in brain cancer incidence rates in relation to mobile phone use in England. Bioelectromagnetics. 2011;32(5):334-339.

10. Kohler BA, Ward E, Mccarthy BJ, et al. Annual report to the nation on the status of cancer, 1975-2007, featuring tumors of the brain and other nervous system. J Natl Cancer Inst. 2011;103(9):714-736.

11. Chapman S, Azizi L, Luo Q, Sitas F. Has the incidence of brain cancer risen in Australia since the introduction of mobile phones 29 years ago? Cancer Epidemiol. 2016;42:199-205.

12. Fitzsimmons D, Osmond C, George S, Johnson CD. Trends in stomach and pancreatic cancer incidence and mortality in England and Wales, 1951-2000. Br J Surg. 2007;94(9):1162-1171.
13. Luo J, Adami HO, Reilly M, Ekbom A, Nordenvall C, Ye W. Interpreting trends of pancreatic cancer incidence and mortality: a nation-wide study in Sweden (1960-2003). Cancer Causes Control. 2008;19(1):89-96.

14. von Hahn T, Ciesek S, Wegener G, et al. Epidemiological trends in incidence and mortality of hepatobiliary cancers in Germany. Scand J Gastroenterol. 2011;46(9):1092-1098.

15. Ludvigsson JF, Andersson E, Ekbom A, et al. External review and validation of the Swedish national inpatient register. BMC Public Health. 2011;11:450.

16. Brooke HL, Talbäck M, Hörnblad J, et al. The Swedish cause of death register. Eur J Epidemiol. 2017;32(9):765-773.

17. Engholm G, Ferlay J, Christensen N, et al. NORDCAN--a Nordic tool for cancer information, planning, quality control and research. Acta Oncol. 2010;49(5):725-736.

18. Larjavaara S, Haapasalo H, Sankila R, Helén P, Auvinen A. Is the incidence of meningiomas underestimated? A regional survey. $\mathrm{Br} J$ Cancer. 2008;99(1):182-184.

19. Li XR, Kruchko C, Wu XC, et al. Are benign and borderline brain tumors underreported? J Registry Manag. 2016;43(4):187-194.

20. Gjerstorff ML. The Danish cancer registry. Scand J Public Health. 2011;39(7 Suppl):42-45. 


\section{Supplementary materials}

Table SI List of ICD codes used to identify central nervous system tumor patients in the Inpatient Register and Cause of Death Register

\begin{tabular}{|l|l|l|}
\hline Brain tumors & ICD-9 & ICD-I0 \\
\hline Malignant & 191 & C7I \\
Benign & $225 \mathrm{~A}$ & D33.0-D33.2 \\
Unknown grading & $237 \mathrm{~F}$ & D43.0-D43.2 \\
Other CNS tumors & & \\
Malignant & 192 & C70; C72 \\
Benign & $225 \mathrm{~B} ; 225 \mathrm{C} ; 225 \mathrm{E}$ & D32; D33.3-D33.9; D42 \\
Unknown grading & 237G; 237X & D42; D43.3-D43.9 \\
\hline
\end{tabular}

Table S2 Total number and proportions of patients with a central nervous system tumor diagnosis in the Inpatient Register not found in the Swedish Cancer Register between 1990-2014, by number of hospital discharge diagnosis containing a central nervous system tumor diagnosis

\begin{tabular}{|l|l|l|l|l|}
\hline \multirow{2}{*}{} & \multicolumn{4}{l|}{ Number of hospital discharge diagnosis (\%) } \\
\cline { 2 - 5 } & At least one & At least two & At least three & At least four \\
\hline Brain tumors & $\mathrm{N}=34,612$ & $\mathrm{~N}=23,842$ & $\mathrm{~N}=17,944$ & $\mathrm{~N}=13,445$ \\
All combined & 22.2 & 15.9 & 11.5 & 8.5 \\
Malignant & 18.3 & 13.6 & 9.5 & 7.1 \\
Benign & 35.8 & 26.8 & 21.4 & 16.9 \\
Unknown behavior & 24.0 & 16.7 & 12.5 & 9.2 \\
Other CNS tumors & $\mathrm{N}=24,086$ & $\mathrm{~N}=13,996$ & $\mathrm{~N}=8,332$ & $\mathrm{~N}=4,906$ \\
All combined & 30.7 & 21.5 & 18.3 & 17.4 \\
Malignant & 15.8 & 14.3 & 11.4 & 11.2 \\
Benign & 32.2 & 22.0 & 18.8 & 18.2 \\
Unknown behavior & 31.9 & 22.6 & 19.3 & 17.2 \\
\hline
\end{tabular}

Abbreviation: CNS, central nervous system.

Table S3 Total number and proportion of central nervous system tumor cases in the Inpatient Register not found in the Outpatient Register with a central nervous system tumor diagnosis between 200 I-20I4

\begin{tabular}{|c|c|c|c|c|}
\hline & \multicolumn{2}{|l|}{ Brain tumors } & \multicolumn{2}{|c|}{ Other CNS tumors } \\
\hline & $\begin{array}{l}\text { Not found in } \\
\text { OutpR (\%) }\end{array}$ & Total & $\begin{array}{l}\text { Not found in } \\
\text { OutpR (\%) }\end{array}$ & Total \\
\hline \multicolumn{5}{|l|}{ All ages } \\
\hline Not reported SCR & $804(35.6)$ & 2,255 & $\mathrm{I}, 634(38.6)$ & 4,233 \\
\hline Reported SCR & I,242 (I5.4) & 8,079 & I,492 (I7.I) & 8,721 \\
\hline \multicolumn{5}{|l|}{ Age $0-19$ years } \\
\hline Not reported SCR & $58(19.8)$ & 293 & $31(3 \mid .0)$ & 100 \\
\hline Reported SCR & $4 I(4.4)$ & 891 & $20(11.9)$ & 168 \\
\hline \multicolumn{5}{|l|}{ Age $20-69$ years } \\
\hline Not reported SCR & 466 (32.9) & $1,4 \mid 8$ & $555(25.7)$ & 2,160 \\
\hline Reported SCR & $766(13.0)$ & 5,873 & $644(10.7)$ & 6,011 \\
\hline \multicolumn{5}{|l|}{ Age $70+$ years } \\
\hline Not reported SCR & $280(5 \mid .5)$ & 544 & $\mathrm{I}, 048(53.1)$ & 1,973 \\
\hline Reported SCR & $435(34.1)$ & 1,274 & $828(32.6)$ & 2,542 \\
\hline
\end{tabular}

Notes: Only patients surviving at least one year have been included in this analysis.

Abbreviations: CNS, central nervous system; SCR, Swedish Cancer Register; OutpR, Outpatient Register. 


\section{Publish your work in this journal}

Clinical Epidemiology is an international, peer-reviewed, open access, online journal focusing on disease and drug epidemiology, identification of risk factors and screening procedures to develop optimal preventative initiatives and programs. Specific topics include: diagnosis, prognosis, treatment, screening, prevention, risk factor modification,

Submit your manuscript here: https://www.dovepress.com/clinical-epidemiology-journal systematic reviews, risk and safety of medical interventions, epidemiology and biostatistical methods, and evaluation of guidelines, translational medicine, health policies and economic evaluations. The manuscript management system is completely online and includes a very quick and fair peer-review system, which is all easy to use. 